\title{
Correction to: Elasmobranchs of the Mexican Caribbean: biodiversity and conservation status
}

María del Pilar Blanco-Parra
Carlos Alberto Niño-Torres

Published online: 18 January 2022

(C) Springer Nature B.V. 2022

Correction to: Environ Biol Fish (2022) 105(1):151-165 https://doi.org/10.1007/s10641-021-01203-7

The original published version of Table 1 contained errors. The corrected Table 1 is as follows:

The original article can be found online at https://doi.org/ 10.1007/s10641-021-01203-7.

M. del Pilar Blanco-Parra

Consejo Nacional de Ciencia y Tecnología, Av. Insurgentes Sur 1582, Col. Crédito Constructor, Alcaldía Benito Juárez, C.P. 03940 Mexico City, Mexico e-mail: mpblancop@gmail.com

M. del Pilar Blanco-Parra · C. A. Niño-Torres División en Desarrollo Sustentable, Universidad de Quintana Roo, Blvd. Bahía S/N Esq. Ignacio Comonfort, Col. Del Bosque, 77019 Chetumal, Quintana Roo, Mexico

M. del Pilar Blanco-Parra · C. A. Niño-Torres $(\bowtie)$ Fundación Internacional Para La Naturaleza y La sustentabilidad (FINS), Calle Larún M75 L4, Andara, 77014 Chetumal, Quintana Roo, Mexico e-mail: carlosalni@gmail.com 
Table 1 Elasmobranch species for the Mexican Caribbean, number of sources (\# S.). Type of source: BC, organisms deposited in national and international scientific collections or museums; JP, articles in refereed journals; $\mathrm{BCH}$, book or book chapter; TR, corroborated reports of governmental, academic, or non-governmental institutions; FS, species found in the catches of the local artisanal fishery; and OD, species reported in online database. Confirmation: confirmed (C) and non-confirmed (NC) and conservation status (NE not evalu- ate, DD data deficient, LC least concern, NT near threatened, VU vulnerable, EN endangered, CR critically endangered, A threatened, $\mathrm{P}$ near extinction, Pr special protection) based on the International Union for Conservation of Nature's Red List of Threatened Species (IUCN), Convention on International Trade in Endangered Species of Wild Fauna and Flora (CITES) and Mexican Law (NOM-059-SEMARNAT). Nomenclature based on Fricke et al. (2021), Ebert et al. (2021), and Last et al. (2016)

\begin{tabular}{|c|c|c|c|c|c|c|c|c|}
\hline ORDER & FAMILY & SPECIES & $\# \mathrm{~S}$ & $\begin{array}{l}\text { Type of source cat- } \\
\text { egories }\end{array}$ & Conf & IUCN & CITES & NOM-059 \\
\hline \multirow[t]{2}{*}{ Hexanchiformes } & Hexanchidae & $\begin{array}{l}\text { Hexanchus nakamu- } \\
\quad \text { rai Teng, } 1962\end{array}$ & 3 & FS:1, JP:1, TR:1 & $\mathrm{C}$ & NT & & \\
\hline & & $\begin{array}{l}\text { Hexanchus griseus } \\
\text { (Bonnaterre, 1788) }\end{array}$ & 2 & BCH:1, JP:1 & $\mathrm{C}$ & NT & & \\
\hline \multirow[t]{14}{*}{ Squaliformes } & Echinorhinidae & $\begin{array}{r}\text { Echinorhinus brucus } \\
\text { (Bonnaterre, 1788) }\end{array}$ & 3 & BC:1, JP:1, OD:1 & $\mathrm{C}$ & EN & & \\
\hline & Squalidae & $\begin{array}{l}\text { Cirrhigaleus asper } \\
\text { (Merrett, 1973) }\end{array}$ & 2 & BCH:1, TR:1 & $\mathrm{C}$ & DD & & \\
\hline & & $\begin{array}{l}\text { Squalus cubensis } \\
\text { Howell-Rivero, } \\
1936\end{array}$ & 3 & BCH:1, JP:2 & $\mathrm{C}$ & $\mathrm{LC}$ & & \\
\hline & & $\begin{array}{l}\text { Centrophorus granu- } \\
\text { losus (Bloch \& } \\
\text { Schneider 1801) }\end{array}$ & 4 & $\begin{array}{l}\text { BC:1, BCH:1, JP:1, } \\
\text { OD:1 }\end{array}$ & $\mathrm{C}$ & EN & & \\
\hline & & $\begin{array}{c}\text { Centrophorus sp. Del } \\
\text { Moral et al., 2016* }\end{array}$ & 1 & JP:1 & $\mathrm{NC}$ & $\mathrm{NE}$ & & \\
\hline & & $\begin{array}{l}\text { Centrophorus uyato } \\
\text { (Rafinesque, 1810) }\end{array}$ & 3 & BC:1, JP:1, OD:1 & $\mathrm{C}$ & EN & & \\
\hline & Etmopteridae & $\begin{array}{l}\text { Etmopterus bigelowi } \\
\text { Shirai \& Tachikawa, } \\
1993\end{array}$ & 1 & BCH: 1 & $\mathrm{NC}$ & $\mathrm{LC}$ & & \\
\hline & & $\begin{array}{l}\text { Etmopterus bul- } \\
\text { lisi Bigelow \& } \\
\text { Schroeder, } 1957\end{array}$ & 1 & JP:1 & $\mathrm{C}$ & $\mathrm{LC}$ & & \\
\hline & & $\begin{array}{l}\text { Etmopterus gracili- } \\
\text { spinis Krefft, } 1968\end{array}$ & 1 & BC: 1 & $\mathrm{NC}$ & $\mathrm{LC}$ & & \\
\hline & & $\begin{array}{l}\text { Etmopterus hillianus } \\
\text { (Poey, 1861) }\end{array}$ & 1 & JP: 1 & $\mathrm{C}$ & LC & & \\
\hline & & $\begin{array}{l}\text { Etmopterus schultzi } \\
\text { Bigelow, Schroeder } \\
\text { \& Springer, } 1953\end{array}$ & 2 & JP:1, OD:1 & $\mathrm{C}$ & $\mathrm{LC}$ & & \\
\hline & & $\begin{array}{l}\text { Etmopterus virens } \\
\text { Bigelow, Schroeder } \\
\text { \& Springer, } 1953\end{array}$ & 3 & BCH:1, JP:1, OD:2 & $\mathrm{C}$ & $\mathrm{LC}$ & & \\
\hline & Somniosidae & $\begin{array}{l}\text { Centroscymnus } \\
\text { owstoni Garman, } \\
1906\end{array}$ & 1 & JP:1 & $\mathrm{C}$ & VU & & \\
\hline & Oxynotidae & $\begin{array}{l}\text { Oxynotus caribbaeus } \\
\text { Cervigon, } 1961\end{array}$ & 1 & JP:1 & $\mathrm{C}$ & $\mathrm{LC}$ & & \\
\hline Squatiniformes & Squatinidae & $\begin{array}{l}\text { Squatina dumeril } \\
\text { Lesueur, } 1818\end{array}$ & 3 & BC:1, JP:1, OD:1 & $\mathrm{NC}$ & $\mathrm{LC}$ & & \\
\hline Laminiformes & Alopiidae & $\begin{array}{l}\text { Alopias superciliosus } \\
\quad \text { (Lowe, } 1841)\end{array}$ & 4 & $\begin{array}{l}\text { BCH:1, BC:1, JP:1, } \\
\text { OD: } 1\end{array}$ & $\mathrm{C}$ & VU & II & \\
\hline
\end{tabular}


Table 1 (continued)

\begin{tabular}{|c|c|c|c|c|c|c|c|c|}
\hline ORDER & FAMILY & SPECIES & $\# \mathrm{~S}$ & $\begin{array}{l}\text { Type of source cat- } \\
\text { egories }\end{array}$ & Conf & IUCN & CITES & NOM-059 \\
\hline & Lamnidae & $\begin{array}{l}\text { Isurus oxyrinchus } \\
\text { Rafinesque, } 1810\end{array}$ & 6 & $\begin{array}{l}\text { BCH:2, FS:1, JP:1, } \\
\text { OD:1, TR:1 }\end{array}$ & $\mathrm{C}$ & EN & II & \\
\hline & Cetorhinidae & $\begin{array}{l}\text { Cetorhinus maximus } \\
\text { (Gunnerus, 1765) }\end{array}$ & 1 & BCH:1 & $\mathrm{NC}$ & EN & II & A \\
\hline \multirow[t]{2}{*}{ Orectolobiformes } & Ginglymostomatidae & $\begin{array}{l}\text { Ginglymostoma cir- } \\
\text { ratum (Bonnaterre, } \\
1788 \text { ) }\end{array}$ & 6 & $\begin{array}{l}\text { BCH:2, FS:1, JP:2, } \\
\text { TR:1 }\end{array}$ & $\mathrm{C}$ & VU & & \\
\hline & Rhincodontidae & $\begin{array}{l}\text { Rhincodon typus } \\
\text { Smith, } 1828\end{array}$ & 5 & $\begin{array}{l}\text { BCH:2, FS:1, JP:1, } \\
\text { TR:1 }\end{array}$ & $\mathrm{C}$ & EN & II & A \\
\hline \multirow[t]{17}{*}{ Carcharhiniformes } & Scyliorhinidae & $\begin{array}{l}\text { Galeus arae (Nichols, } \\
\text { 1927) }\end{array}$ & 4 & $\begin{array}{l}\text { JP:1, BCH:1, BC:1, } \\
\text { OD:1 }\end{array}$ & $\mathrm{C}$ & $\mathrm{LC}$ & & \\
\hline & & $\begin{array}{l}\text { Scyliorhinus hespe- } \\
\quad \text { rius Springer, } 1966\end{array}$ & 3 & BC:1, JP:1, OD:1 & $\mathrm{C}$ & $\mathrm{LC}$ & & \\
\hline & & $\begin{array}{l}\text { Scyliorhinus retifer } \\
\text { (Garman, 1881) }\end{array}$ & 4 & $\begin{array}{l}\text { BCH:1, BC:1, JP:1, } \\
\text { OD:1 }\end{array}$ & $\mathrm{C}$ & $\mathrm{LC}$ & & \\
\hline & & $\begin{array}{l}\text { Scyliorhinus meadi } \\
\text { Springer, } 1966\end{array}$ & 3 & BCH:1, JP:1, TR:1 & $\mathrm{C}$ & $\mathrm{LC}$ & & \\
\hline & Triakidae & $\begin{array}{l}\text { Mustelus canis } \\
\quad \text { (Mitchill, 1815) }\end{array}$ & 4 & $\begin{array}{l}\text { BCH:1, FS:1, JP:1, } \\
\text { TR:1 }\end{array}$ & $\mathrm{C}$ & NT & & \\
\hline & & $\begin{array}{l}\text { Mustelus higmani } \\
\text { Springer \& Lowe, } \\
1963\end{array}$ & 3 & BCH:1, FS:1, JP:1 & $\mathrm{C}$ & EN & & \\
\hline & Carcharhinidae & $\begin{array}{l}\text { Carcharhinus acrono- } \\
\text { tus (Poey, 1860) }\end{array}$ & 6 & $\begin{array}{l}\text { BCH:2, FS:1, JP:2, } \\
\text { TR:1 }\end{array}$ & $\mathrm{C}$ & EN & & \\
\hline & & $\begin{array}{l}\text { Carcharhinus altimus } \\
\text { (Springer, 1950) }\end{array}$ & 2 & BCH:1, JP:1 & $\mathrm{C}$ & NT & & \\
\hline & & $\begin{array}{l}\text { Carcharhinus brach- } \\
\text { yurus (Günther, } \\
1870)\end{array}$ & 2 & OD:1, TR:1 & $\mathrm{C}$ & VU & & \\
\hline & & $\begin{array}{l}\text { Carcharhinus brevi- } \\
\text { pinna (Valenci- } \\
\text { ennes, 1839) }\end{array}$ & 5 & $\begin{array}{l}\text { BC: } 1, \text { FS:1, JP:1, } \\
\text { OD:1, TR: } 1\end{array}$ & $\mathrm{C}$ & VU & & \\
\hline & & $\begin{array}{l}\text { Carcharhinus falci- } \\
\text { formis (Müller \& } \\
\text { Henle, 1839) }\end{array}$ & 7 & $\begin{array}{c}\text { BC: } 1, \text { BCH: } 1, \text { FS: } 1 \text {, } \\
\text { JP:2, OD:1, TR: } 1\end{array}$ & $\mathrm{C}$ & VU & II & \\
\hline & & $\begin{array}{l}\text { Carcharhinus leucas } \\
\text { (Valenciennes, } \\
\text { 1839) }\end{array}$ & 7 & $\begin{array}{l}\text { BCH:2, FS:1, JP:3, } \\
\text { TR:1 }\end{array}$ & $\mathrm{C}$ & VU & & \\
\hline & & $\begin{array}{l}\text { Carcharhinus limba- } \\
\text { tus (Valenciennes, } \\
\text { 1839) }\end{array}$ & 7 & $\begin{array}{l}\text { BCH:2, FS:1, JP:3, } \\
\text { TR:1 }\end{array}$ & $\mathrm{C}$ & VU & & \\
\hline & & $\begin{array}{l}\text { Carcharhinus longi- } \\
\text { manus (Poey, 1861) }\end{array}$ & 2 & BCH:1, JP:1 & $\mathrm{C}$ & $\mathrm{CR}$ & II & \\
\hline & & $\begin{array}{l}\text { Carcharhinus obscu- } \\
\text { rus (Lesueur, 1818) }\end{array}$ & 7 & $\begin{array}{l}\text { BCH:2, FS:1, JP:2, } \\
\text { OD:1, TR: } 1\end{array}$ & $\mathrm{C}$ & EN & & \\
\hline & & $\begin{array}{l}\text { Carcharhinus perezi } \\
\text { (Poey, 1876) }\end{array}$ & 8 & $\begin{array}{c}\text { BC:1, BCH:2, FS:1, } \\
\text { JP:2, OD: } 1 \text {, TR: } 1\end{array}$ & $\mathrm{C}$ & EN & & \\
\hline & & $\begin{array}{l}\text { Carcharhinus } \\
\text { plumbeus (Nardo, } \\
\text { 1827) }\end{array}$ & 6 & $\begin{array}{l}\text { BCH:2, FS:1, JP:1, } \\
\text { OD:1, TR:1 }\end{array}$ & $\mathrm{C}$ & EN & & \\
\hline
\end{tabular}


Table 1 (continued)

\begin{tabular}{|c|c|c|c|c|c|c|c|c|}
\hline ORDER & FAMILY & SPECIES & $\# \mathrm{~S}$ & $\begin{array}{l}\text { Type of source cat- } \\
\text { egories }\end{array}$ & Conf & IUCN & CITES & NOM-059 \\
\hline & & $\begin{array}{l}\text { Carcharhinus porosus } \\
\text { (Ranzani, 1839) }\end{array}$ & 4 & BCH:2, JP:2 & $\mathrm{C}$ & CR & & \\
\hline & & $\begin{array}{l}\text { Carcharhinus signa- } \\
\text { tus (Poey, 1868) }\end{array}$ & 2 & JP: 1, TR:1 & $\mathrm{C}$ & EN & & \\
\hline & & $\begin{array}{l}\text { Rhizoprionodon poro- } \\
\text { sus (Poey, 1861) }\end{array}$ & 6 & $\begin{array}{l}\text { BCH:2, FS:1, JP:1, } \\
\text { OD:2 }\end{array}$ & $\mathrm{C}$ & VU & & \\
\hline & & $\begin{array}{l}\text { Rhizoprionodon ter- } \\
\text { raenovae (Richard- } \\
\text { son, } 1837 \text { ) }\end{array}$ & 8 & $\begin{array}{c}\text { BC:1, BCH:1, FS:1, } \\
\text { JP:2, OD:2, TR:1 }\end{array}$ & $\mathrm{C}$ & $\mathrm{LC}$ & & \\
\hline & & $\begin{array}{l}\text { Prionace glauca } \\
\quad \text { (Linnaeus, 1758) }\end{array}$ & 2 & BCH:1, OD: 1 & $\mathrm{C}$ & NT & & \\
\hline & & $\begin{array}{l}\text { Negaprion breviro- } \\
\text { stris (Poey, 1868) }\end{array}$ & 8 & $\begin{array}{c}\text { BC: } 1, \text { BCH:1, FS:1, } \\
\text { JP:2, OD:2, TR:1 }\end{array}$ & $\mathrm{C}$ & $\mathrm{VU}$ & & \\
\hline & Galeocerdidae & $\begin{array}{l}\text { Galeocerdo cuvier } \\
\text { (Perón \& Lesueur, } \\
\text { 1822) }\end{array}$ & 7 & $\begin{array}{l}\text { BCH:2, FS:1, JP:3, } \\
\text { TR:1 }\end{array}$ & $\mathrm{C}$ & NT & & \\
\hline & Sphyrnidae & $\begin{array}{l}\text { Sphyrna lewini (Grif- } \\
\text { fith \& Smith, 1834) }\end{array}$ & 4 & $\begin{array}{l}\text { BCH:1, FS:1, JP:1, } \\
\text { TR:1 }\end{array}$ & $\mathrm{C}$ & CR & II & \\
\hline & & $\begin{array}{l}\text { Sphyrna mokarran } \\
\text { (Rüppell, 1837) }\end{array}$ & 6 & $\begin{array}{l}\text { BCH:1, FS:1, JP:2, } \\
\text { OD:1, TR:1 }\end{array}$ & $\mathrm{C}$ & $\mathrm{CR}$ & II & \\
\hline & & $\begin{array}{l}\text { Sphyrna tiburo (Lin- } \\
\text { naeus, 1758) }\end{array}$ & 7 & $\begin{array}{l}\text { BCH:2, FS:1, JP:3, } \\
\text { TR:1 }\end{array}$ & $\mathrm{C}$ & EN & & \\
\hline \multirow[t]{4}{*}{ Rhinopristiformes } & Pristidae & $\begin{array}{l}\text { Pristis pectinata } \\
\text { Latham, } 1794\end{array}$ & 5 & BCH:2, JP:2, TR:1 & $\mathrm{C}$ & $\mathrm{CR}$ & I & $\mathrm{P}$ \\
\hline & & $\begin{array}{l}\text { Pristis pristis (Lin- } \\
\text { naeus, 1758) }\end{array}$ & 2 & BC:1, JP:1 & $\mathrm{C}$ & CR & I & $\mathrm{P}$ \\
\hline & Rhinobatidae & $\begin{array}{l}\text { Pseudobatos len- } \\
\text { tiginosus (Garman, } \\
\text { 1880) }\end{array}$ & 6 & $\begin{array}{l}\text { BC: } 1, \text { BCH:1, FS:1, } \\
\text { JP:1, OD:2 }\end{array}$ & $\mathrm{C}$ & VU & & \\
\hline & & $\begin{array}{l}\text { Pseudobatos percel- } \\
\text { lens (Walbaum, } \\
\text { 1792) }\end{array}$ & 3 & BC:1, JP:2 & $\mathrm{C}$ & EN & & \\
\hline \multirow[t]{3}{*}{ Torpediniformes } & Narcinidae & $\begin{array}{l}\text { Narcine bancroftii } \\
\text { (Griffith \& Smith, } \\
\text { 1834) }\end{array}$ & 4 & $\begin{array}{l}\text { BC: } 1, \text { BCH:1, FS:1, } \\
\text { JP: } 1\end{array}$ & $\mathrm{C}$ & $\mathrm{LC}$ & & \\
\hline & & $\begin{array}{l}\text { Narcine brasiliensis } \\
\quad(\text { Olfers, 1831) }\end{array}$ & 4 & BC:2, JP:2, & $\mathrm{C}$ & NT & & \\
\hline & Torpedinidae & $\begin{array}{l}\text { Torpedo andersoni } \\
\text { Bullis, } 1962\end{array}$ & 2 & BCH:1, JP:1 & $\mathrm{C}$ & $\mathrm{LC}$ & & \\
\hline \multirow[t]{4}{*}{ Rajiformes } & Arhynchobatidae & $\begin{array}{l}\text { Pseudoraja fis- } \\
\text { cheri Bigelow \& } \\
\text { Schroeder, } 1954\end{array}$ & 5 & $\begin{array}{l}\text { BC:1, BCH:1, JP:1, } \\
\text { OD:2 }\end{array}$ & $\mathrm{C}$ & $\mathrm{LC}$ & & \\
\hline & Rajidae & $\begin{array}{l}\text { Cruriraja poeyi } \\
\text { Bigelow \& } \\
\text { Schroeder, } 1948\end{array}$ & 1 & BCH:1 & $\mathrm{NC}$ & $\mathrm{LC}$ & & \\
\hline & & $\begin{array}{l}\text { Cruriraja rugosa } \\
\text { Bigelow \& } \\
\text { Schroeder, } 1958\end{array}$ & 1 & BCH:1 & $\mathrm{NC}$ & $\mathrm{LC}$ & & \\
\hline & & $\begin{array}{l}\text { Breviraja spi- } \\
\text { nosa } \text { Bigelow \& } \\
\text { Schroeder } 1950\end{array}$ & 3 & BC:1, OD:2 & $\mathrm{C}$ & LC & & \\
\hline
\end{tabular}


Table 1 (continued)

\begin{tabular}{|c|c|c|c|c|c|c|c|}
\hline ORDER & FAMILY & SPECIES & \# S & $\begin{array}{l}\text { Type of source cat- } \\
\text { egories }\end{array}$ & Conf & IUCN CITES & NOM-059 \\
\hline & & $\begin{array}{l}\text { Dipturus olseni } \\
\quad \text { (Bigelow \& } \\
\text { Schroeder, 1951) }\end{array}$ & 2 & BC:1, JP:1 & $\mathrm{C}$ & $\mathrm{LC}$ & \\
\hline & & $\begin{array}{l}\text { Dipturus teevani } \\
\text { (Bigelow \& } \\
\text { Schroeder 1951) }\end{array}$ & 1 & BCH:1 & $\mathrm{NC}$ & $\mathrm{LC}$ & \\
\hline & & $\begin{array}{l}\text { Fenestraja ishiy- } \\
\text { amai (Bigelow \& } \\
\text { Schroeder, 1962) }\end{array}$ & 2 & BC:1, JP:1 & $\mathrm{C}$ & $\mathrm{LC}$ & \\
\hline & & $\begin{array}{l}\text { Fenestraja plutonia } \\
\quad \text { (Garman, 1881) }\end{array}$ & 2 & BC:1, JP:1 & $\mathrm{C}$ & $\mathrm{LC}$ & \\
\hline & & $\begin{array}{l}\text { Fenestraja sinusmexi- } \\
\text { canus (Bigelow \& } \\
\text { Schroeder, 1950) }\end{array}$ & 2 & BC:1, JP:1 & $\mathrm{C}$ & $\mathrm{LC}$ & \\
\hline & & $\begin{array}{l}\text { Leucoraja garmani } \\
\text { (Whitley, 1939) }\end{array}$ & 6 & $\mathrm{BC}: 2, \mathrm{JP}: 2, \mathrm{OD}: 2$ & $\mathrm{C}$ & $\mathrm{LC}$ & \\
\hline & & $\begin{array}{l}\text { Leucoraja yucatan- } \\
\text { ensis (Bigelow \& } \\
\text { Schroeder, 1950) }\end{array}$ & 6 & $\begin{array}{l}\text { BC:1, BCH:1, JP:2, } \\
\text { OD:2 }\end{array}$ & $\mathrm{C}$ & $\mathrm{LC}$ & \\
\hline & & $\begin{array}{l}\text { Rajella fuliginea } \\
\text { (Bigelow \& } \\
\text { Schroeder, 1954) }\end{array}$ & 1 & BCH:1 & $\mathrm{NC}$ & $\mathrm{LC}$ & \\
\hline & & $\begin{array}{l}\text { Rostroraja ackleyi } \\
\quad \text { (Garman, 1881) }\end{array}$ & 3 & BC:1, BCH:1, JP:1 & $\mathrm{C}$ & $\mathrm{LC}$ & \\
\hline & & $\begin{array}{l}\text { Rostroraja texana } \\
\quad(\text { Chandler, 1921) }\end{array}$ & 5 & $\begin{array}{l}\text { BC:1, BCH:1, JP:2, } \\
\text { OD: } 1\end{array}$ & $\mathrm{C}$ & $\mathrm{LC}$ & \\
\hline \multirow[t]{10}{*}{ Myliobatiformes } & Urotrygonidae & $\begin{array}{l}\text { Urobatis jamaicensis } \\
\quad \text { (Cuvier, 1817) }\end{array}$ & 7 & $\begin{array}{l}\text { BC:2, BCH:1, FS:1, } \\
\text { JP:2, OD: } 1\end{array}$ & $\mathrm{C}$ & $\mathrm{LC}$ & \\
\hline & Dasyatidae & $\begin{array}{l}\text { Hypanus americanus } \\
\text { (Hildebrand \& } \\
\text { Schroeder, 1928) }\end{array}$ & 6 & $\begin{array}{l}\text { BC:2, FS:1, JP:2, } \\
\text { OD:1, TR:1 }\end{array}$ & $\mathrm{C}$ & NT & \\
\hline & & $\begin{array}{l}\text { Hypanus say (Lesu- } \\
\text { eur, 1817) }\end{array}$ & 3 & FS:1, JP:1, TR:1 & $\mathrm{C}$ & NT & \\
\hline & & $\begin{array}{l}\text { Hypanus sabinus } \\
\text { (Lesueur, 1824) }\end{array}$ & 2 & JP:1, OD:1 & $\mathrm{C}$ & $\mathrm{LC}$ & \\
\hline & & $\begin{array}{l}\text { Hypanus guttatus } \\
\text { (Bloch \& Schneider, } \\
\text { 1801) }\end{array}$ & 4 & $\begin{array}{l}\text { BC:1, BCH:1, FS:1, } \\
\text { OD: } 1\end{array}$ & $\mathrm{C}$ & NT & \\
\hline & & $\begin{array}{l}\text { Pteroplatytrygon vio- } \\
\text { lacea (Bonaparte, } \\
\text { 1832) }\end{array}$ & 1 & BCH:1 & $\mathrm{NC}$ & $\mathrm{LC}$ & \\
\hline & Potamotrygonidae & $\begin{array}{l}\text { Styracura schmardae } \\
\text { (Werner, 1904) }\end{array}$ & 9 & $\begin{array}{c}\text { BC:1, BCH:2, FS:1, } \\
\text { JP:2, OD:2, TR: } 1\end{array}$ & $\mathrm{C}$ & EN & \\
\hline & Gymnuridae & $\begin{array}{l}\text { Gymnura micrura } \\
\text { (Bloch \& Schneider, } \\
\text { 1801) }\end{array}$ & 6 & $\begin{array}{l}\text { BC: } 1, \text { BCH: } 1, \text { FS: } 1 \text {, } \\
\text { JP: } 1, \text { OD:2 }\end{array}$ & $\mathrm{C}$ & NT & \\
\hline & Aetobatidae & $\begin{array}{l}\text { Aetobatus narinari } \\
\quad \text { (Eupharasen, 1790) }\end{array}$ & 5 & $\begin{array}{l}\text { BCH:1, FS:1, JP:1, } \\
\text { OD:1, TR: } 1\end{array}$ & $\mathrm{C}$ & EN & \\
\hline & Myliobatidae & $\begin{array}{l}\text { Myliobatis goodei } \\
\text { Garman, } 1885\end{array}$ & 1 & BCH:1 & $\mathrm{NC}$ & VU & \\
\hline
\end{tabular}


Table 1 (continued)

\begin{tabular}{|c|c|c|c|c|c|c|c|c|}
\hline ORDER & FAMILY & SPECIES & $\# \mathrm{~S}$ & $\begin{array}{l}\text { Type of source cat- } \\
\text { egories }\end{array}$ & Conf & IUCN & CITES & NOM-059 \\
\hline & \multirow[t]{2}{*}{ Rhinopteridae } & $\begin{array}{l}\text { Rhinoptera bonasus } \\
\text { (Mitchill, 1815) }\end{array}$ & 3 & BCH:1, FS:1, OD:1 & $\mathrm{C}$ & VU & & \\
\hline & & $\begin{array}{l}\text { Rhinoptera brasilien- } \\
\text { sis Müller, } 1836\end{array}$ & 1 & BCH: 1 & $\mathrm{NC}$ & VU & & \\
\hline & \multirow[t]{3}{*}{ Mobulidae } & $\begin{array}{l}\text { Mobula hypostoma } \\
\text { (Bancroft, 1831) }\end{array}$ & 3 & FS:1, JP:1, OD:1 & $\mathrm{C}$ & EN & II & $\operatorname{Pr}$ \\
\hline & & $\begin{array}{l}\text { Mobula birostris } \\
\text { (Walbaum, 1792) }\end{array}$ & 5 & $\begin{array}{l}\text { BCH:1, FS:1, JP:2, } \\
\text { OD:1 }\end{array}$ & $\mathrm{C}$ & EN & II & $\operatorname{Pr}$ \\
\hline & & $\begin{array}{l}\text { Mobula cf. birostris } \\
\text { Hinojosa-Alvarez } \\
\text { et al. 2016* }\end{array}$ & 2 & FS:1, JP:1 & $\mathrm{NC}$ & EN & II & Pr \\
\hline
\end{tabular}

*Authors report evidence for new species but there are not yet described

Publisher's note Springer Nature remains neutral with regard to jurisdictional claims in published maps and institutional affiliations. 